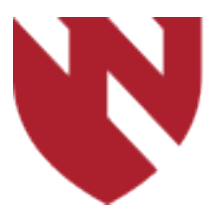

October 2021

\title{
Parkinsonism-Hyperpyrexia Syndrome Secondary to Deep Brain Stimulation Withdrawal Treated with High Doses of Levodopa- Carbidopa: A Rare Life-Threatening Emergency
}

\author{
Daniel Crespo \\ University of Nebraska Medical Center \\ Kiel Woodward \\ University of Nebraska Medical Center \\ Miguel Situ-Kcomt \\ University of Nebraska Medical Center
}

Tell us how you used this information in this short survey.

Follow this and additional works at: https://digitalcommons.unmc.edu/gmerj

Part of the Higher Education Commons, and the Medicine and Health Sciences Commons

\section{Recommended Citation}

Crespo, D., Woodward, K., , Situ-Kcomt, M. Parkinsonism-Hyperpyrexia Syndrome Secondary to Deep Brain Stimulation Withdrawal Treated with High Doses of Levodopa-Carbidopa: A Rare Life-Threatening Emergency. Graduate Medical Education Research Journal. 2021 Oct 04; 3(1).

https://digitalcommons.unmc.edu/gmerj/vol3/iss1/37

This Conference Proceeding is brought to you for free and open access by DigitalCommons@UNMC. It has been accepted for inclusion in Graduate Medical Education Research Journal by an authorized editor of DigitalCommons@UNMC.For more information, please contact digitalcommons@unmc.edu. 


\section{Parkinsonism-Hyperpyrexia Syndrome Secondary to Deep Brain Stimulation}

Withdrawal Treated with High Doses of Levodopa-Carbidopa: A Rare LifeThreatening Emergency

Creative Commons License

(ब) $\Theta \Theta$

This work is licensed under a Creative Commons Attribution-Noncommercial-No Derivative Works 4.0 License. 
attempted but he had an anaphylactic reaction. He was ultimately treated with IV steroids and IVIG with poor recovery. Consent was obtained.

Conclusions: VZV-associated LETM is an unusual complication of treated HZ. Although there is no standard treatment, acyclovir, IVIG and plasma exchange are used as potential treatments, ultimately with poor prognosis. Negative imaging long after symptom onset emphasizes the importance of a good neurologic exam and repeat imaging for diagnosis.

https://doi.org/10.32873/unmc.dc.gmerj.3.1.020

\section{Parkinsonism-Hyperpyrexia Syndrome Secondary to Deep Brain Stimulation Withdrawal Treated with High Doses of Levodopa-Carbidopa: a Rare Life-Threatening Emergency} Daniel Crespo ${ }^{1}$, Kiel Woodward ${ }^{1}$, Miguel Situ-Kcomt ${ }^{1}$

${ }^{1}$ University of Nebraska Medical Center, College of Medicine, Department of Neurological Sciences

Mentor: Miguel Situ-Kcomt

Program: Neurology

Type: Case Report

Background: Parkinsonism-HyperpyrexiaSyndrome (PHS) is an emergency mimicking Neuroleptic-Malignant-Syndrome (NMS) and presents with rigidity, hyperpyrexia, increased creatine phosphokinase (CPK), altered consciousness, autonomic instability. Abrupt discontinuation of dopaminergic therapy in a patient with Parkinson's disease (PD) is the main mechanism causing PHS. This has been generally reported with the sudden interruption of enteral administration of medications but has been anecdotally reported in deep brain stimulation (DBS) withdrawal. Pathophysiology of PHS triggered by DBS discontinuation is poorly understood.

Case: This 56-year-old male had a past medical history of 20 years of Parkinson's disease (PD) status post bilateral STN-DBS 12 years prior and baseline asymmetric tremor, motor fluctuations with dyskinesias, hallucinations, gait imbalance with a Unified PD Rating Scale (UPDRS) of 61. His antiparkinsonian medication regimen consisted of carbidopa-levodopa, ropinirole, trihexyphenidyl, and rivastigmine. He underwent removal of his Implantable Pulse Generator (IPG) due to soft tissue infection. He then presented severe worsening of parkinsonism with a UPDRS of 96. Neurological exam showed severe dysarthria, persistent head rotational tremor, severe tremor at the bilateral elbow and wrist joints, and moderate tremor at the bilateral ankles. Severe cogwheeling rigidity was present in all extremities.

Although already on adequate directed antibiotics for the infection, he continued to have fevers (102.2 F), hypertension (223 mmHg of systolic blood pressure), tachycardia (heart rate of 150's), and a CPK above 5000. He developed severe dysphagia which prevented him from swallowing medications. After placement of a Nasogastric tube, levodopa-carbidopa dose was restarted and tripled. His tremors slowly improved, dysautonomia resolved, CPK levels normalized.

Conclusions: PHS secondary to DBSwithdrawal is a rare and life-threatening condition occurring upon abrupt DBS cessation. We present a case of PHS in the setting of DBS-withdrawal secondary to IPG infection and removal that resolved with aggressive dopaminergic medication regimen without re-implantation of IPG. Ultimately, DBS restoration is the definitive treatment.

https://doi.org/10.32873/unmc.dc.gmerj.3.1.031

\section{Bilateral Limb-Shaking Transient Ischemic Attack Secondary to Bilateral Anterior Cerebral Artery Stenosis and Right A3 Thrombosis \\ Daniel Crespo ${ }^{1}$, Michael Pichler ${ }^{1}$}

${ }^{1}$ University of Nebraska Medical Center, College of Medicine, Department of Neurological Sciences

Mentor: Michael Pichler

Program: Neurology

Type: Case Report

Background: Limb-shaking Transient Ischemic Attack (TIA) is a very rare presentation of cerebral arterial occlusion. It presents as involuntary abnormal movements that occur in one or more limbs usually triggered by orthostasis and cerebral hypoperfusion. This has been described in unilateral or bilateral carotid artery occlusions but to the best of our knowledge, this is the first case of Limb-Shaking TIA secondary to bilateral anterior cerebral artery (ACA) occlusions.

Case: This is a 76-year-old male with significant cardiovascular comorbidities who presented with three months of recurrent falls preceded by 30 seconds of weakness and shaking of bilateral legs. He did not have any prodromal symptoms or loss of awareness associated with these episodes that occurred several times per day. After a few minutes of being in the ground, he would regain complete strength and would be able to stand up independently. He was found to have orthostatic hypotension during these episodes. Vascular imaging showed a complex anatomy of the anterior cerebral arteries, with fusiform dilation of the right A2 segment, a thrombosed right A3 segment and stenosed left ACA. MRI of the brain did not show diffusion restriction consistent with ischemic injury. He was also found to have lumbar spinal stenosis which was thought to contribute to the weakness. He was started on dual antiplatelet therapy and high intensity statins. His symptoms responded to hydration and careful ambulation with compression stockings.

Conclusion: Bilateral limb-shaking TIAs are a very unusual presentation of cerebral hypoperfusion. They are often misdiagnosed as focal seizures with preserved awareness or even movement disorders such as myoclonus. It is important to identify triggers such as orthostatic hypotension that can be often reproduced at bedside. Management involves careful blood pressure control, avoidance of precipitating factors and consideration of revascularization if applicable.

https://doi.org/10.32873/unmc.dc.gmerj.3.1.019 